\title{
HETEROGENEITY ASSESSMENT BASED ON AVERAGE VARIATIONS OF MORPHOLOGICAL TORTUOSITY FOR COMPLEX POROUS STRUCTURES CHARACTERIZATION
}

\author{
Johan Chaniot ${ }^{1,2}$, Maxime Moreaud ${ }^{\bowtie, 1,3}$, LoḮ SorbieR ${ }^{1}$, Dominique Jeulin ${ }^{3}$, \\ JeAN-MARIE BeCKeR ${ }^{2}$ AND ThIERRY FOURNEL ${ }^{2}$
}

${ }^{1}$ IFP Energies nouvelles, Rond-point de l'échangeur de Solaize, BP 3, 69360 Solaize, France, ${ }^{2}$ Universi-té de Lyon, Université Jean Monnet de Saint-Etienne, CNRS UMR 5516, Laboratoire Hubert Curien, F42000 Saint-Etienne, France, ${ }^{3}$ MINES ParisTech, PSL-Research University, CMM, 35 rue Saint Honoré, 77305 Fontainebleau, France

e-mail: chaniot.johan@gmail.com, maxime.moreaud@ifpen.fr, loic.sorbier@ifpen.fr, jeanmarie.becker@univst-etienne.fr, dominique.jeulin@mines-paristech.fr, fournel@univ-st-etienne.fr

(Received March 12, 2020; revised May 19, 2020; accepted May 23, 2020)

\begin{abstract}
Morphological characterization of porous media is of paramount interest, mainly due to the connections between their physicochemical properties and their porous microstructure geometry. Heterogeneity can be seen as a geometric characteristic of porous microstructures. In this paper, two novel topological descriptors are proposed, based on the M-tortuosity formalism. Using the concept of geometric tortuosity or morphological tortuosity, a first operator is defined, the H-tortuosity. It estimates the average variations of the morphological tortuosity as a function of the scale, based on Monte Carlo method and assessing the heterogeneity of porous networks. The second descriptor is an extension, named the H-tortuosity-by-iterativeerosions, taking into account different percolating particle sizes. These two topological operators are applied on Cox multi-scale Boolean models, to validate their behaviors and to highlight their discriminative power.
\end{abstract}

Keywords: geodesic distance transform, heterogeneity, Monte Carlo algorithms, morphological tortuosity, multi-scale porous network.

\section{INTRODUCTION}

Porous media are of paramount interest for various applications in fields as diverse as heterogeneous catalysis (Raybaud and Toulhoat, 2013), energy research (Neumann et al., 2019) and pharmaceutical sciences (Barman, 2019). Industrial processes require increasingly high performance of materials, which have consequently a complex microstructure. Morphological characterization becomes more and more crucial, mostly because of the connections between use properties of porous media and their microstructure geometry (Dullien, 1979). Numerical description of the morphology of such porous structures is made possible thanks to the technological progress in the last decades, both in terms of imaging devices and computing capacity (Adler, 1992). Therefore, characterizing such media to predict their behavior, by means of numerical operators named descriptors, is still a current challenge. Moreover, classical descriptors are not sufficient for some specific applications. Consequently, new numerical descriptors have to be proposed to overcome this limit.

The concept of tortuosity is one of the foremost descriptor of materials (Carman, 1937; Clennell, 1997; Ghanbarian et al., 2013a), closely linked to percolation theory (Balberg et al., 1984; Newman and Ziff, 2001; Grujicic et al., 2004; Jeulin and Moreaud, 2006; Ghanbarian et al., 2013b) and constrictivity concept (Petersen, 1958; Van Brakel and Heertjes, 1974; Holzer et al., 2013; Bini et al., 2019; Neumann et $a l ., 2019)$. This paper focuses on a specific definition of the concept of morphological tortuosity (Decker et al., 1998; Peyrega and Jeulin, 2013); ratio of the geodesic distance (Lantuéjoul and Beucher, 1981) to the Euclidean distance between two distinct points of the porous network. Chaniot et al. (2019) have defined topological descriptors, named $M$-tortuosity and $M$ tortuosity-by-iterative-erosions, of which the aim is to assess the morphological tortuosity of the overall porous microstructure, and to extend this definition to take into account different probe sizes (Vogel, 2002; Ohser et al., 2012), respectively. These approaches describe the global porous network, but are not focused on the local features.

Heterogeneity is a major characteristic of porous media (Hollewand and Gladden, 1995; Rigby and Gladden, 1996; Adler and Thovert, 1998). This is a common feature of the microstructure of industrial and natural porous media. Indeed, different works in distinct fields deal with the concept of heterogeneity over various physical phenomena (Graham, 1957; 
Warren and Price, 1961; Jeulin, 1993; Karimpouli and Tahmasebi, 2019). From a geometrical viewpoint, heterogeneity can be seen of several ways. Hereafter, heterogeneity is directly related to aggregation as in Jeulin (2010), and is then directly connected to local variations of porosity. Solid aggregates, of higher density, induce heterogeneity and influence the structure topology.

The question we address in this paper is: is it possible to use the M-tortuosity formalism to assess the variation of the morphological tortuosity with the spatial scale? Indeed, the fact that morphological tortuosity is scale-dependent allows a numerical characterization of the microstructure's heterogeneity, and in particular its topology. Our goal is to quantify the geometric heterogeneity by assessing its impact over the morphological tortuosity.

First, notations are given and, the distance transform and the geodesic distance transform are defined. These transforms, quantifying the paths by their length, are at the very basis of the following descriptor definitions. Secondly, as a reminder, the $M$-tortuosity and the $M$ tortuosity-by-iterative-erosions estimators are defined, and their specificities are discussed. The third part presents the deterministic definitions of the $\mathrm{H}$ tortuosity, followed by its estimator, characterizing a certain scale heterogeneity. An extension is proposed, the H-tortuosity-by-iterative-erosions, similarly to Chaniot et al. (2019), which considers various probe sizes in order to promote bottleneck effects. Finally, their behavior is analyzed on Cox multi-scale Boolean models (Jeulin, 2010), evidencing their discriminative power.

\section{METHODS}

\section{NOTATIONS}

\section{Overall notations}

We mainly adopt the notations used in Criminisi et al. (2010) and Lohou and Bertrand (2005).

Let $I$ be a binary function, $I: \mathbb{R}^{3} \rightarrow\{0,1\}$. Feature points are defined by the set $X=\left\{x \in \mathbb{R}^{3} ; I(x)=1\right\}$, where $X$ is a bounded set. The complementary set of $X$ is $X^{c}=\left\{x \in \mathbb{R}^{3} ; I(x)=0\right\}$. Let $\mathfrak{I}$ be a bounded subset of $\mathbb{R}^{3}$, defined as a convex subset such that $X \subset \mathfrak{I}$, and $\partial \mathfrak{I}$ the boundaries of $\mathfrak{I}$. Practically, due to the computer architecture, $\mathfrak{I}$ will be a cube. Let $\mathbb{I}$ be a 3D binary image defined by $I$ and $\mathfrak{I}, \mathbb{I}: \mathfrak{I} \rightarrow\{0,1\}$. In other words, $\mathbb{I}$ is an observation window of $I$ such that $X$ is completely included in that window. These notations are illustrated in Fig. 1. Let $c \in \mathbb{R}^{3}$ be the center of mass of $X$. Let $S=\left\{p_{i}\right\}_{i \in \llbracket 0, N-1 \rrbracket}$ be a set of
$N$ distinct sampled points such that $\forall i \in[0, N-1 \rrbracket$, $p_{i} \in X$.

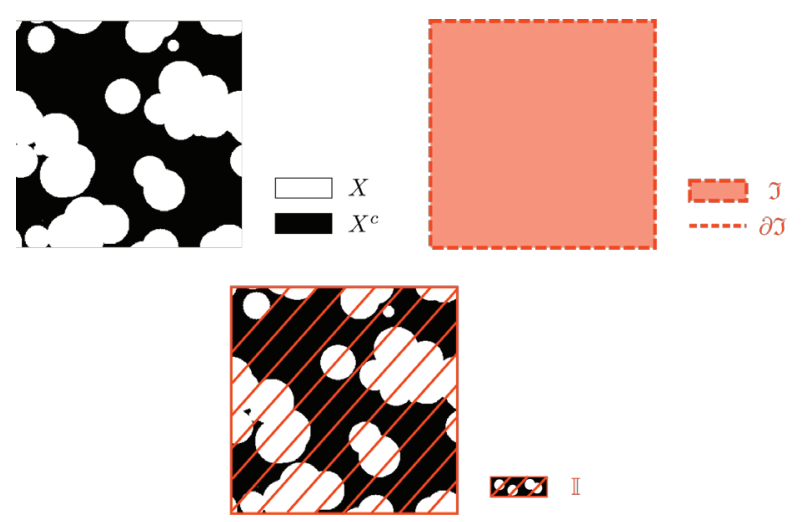

Fig. 1: Illustration of the notations used: $X$ the feature subset, $X^{c}$ its complementary set, $\mathfrak{I}$ a convex subset such as $X \subset \mathfrak{I}, \partial \mathfrak{I}$ its boundaries, and the image $\mathbb{I}$.

In this article, when Boolean models are considered, as below in the Results section, $X$ represents the interconnected porous microstructure, the complementary set of the grains union, and $X^{c}$ the solid phase, the grains union, both constrained by $\mathfrak{I}$; $X^{c}$ is different from the union of grains.

\section{Distance Transform}

The distance transform (Rosenfeld and Pfaltz, 1968; Borgefors, 1986) is a numerical operation which aims to assess the Euclidean distance between a set of points $S^{c}$ and $S$, named hereafter the source points set and $S^{c}$ its complementary set. For each $x \in S^{c}$, the distance transform $D(., S)$ from $S$ is defined as

$$
D(x, S)=\min _{\left\{x^{\prime} \in S\right\}} d\left(x, x^{\prime}\right)
$$

with,

$$
d(x, y)=\inf _{\Gamma \in \gamma_{x, y}} \int_{y}^{x} d s
$$

where $\gamma_{x, y}$ is the set of all possible paths in $\mathbb{R}^{3}$, between $y$, starting point, and $x$, ending point. $\Gamma$ is one of these paths, and $s \in[0, L(\Gamma)]$ its curvilinear abscissa, with $L(\Gamma)$ the length of the path $\Gamma$.

$D(S)$ is the 3D distance map and, $\forall x \in X, D(x, S)$ is the distance transform value at point $x$ from $S$. In the specific case of a singleton, $S=\{y\}, D(x, y)$ stands for $D(x,\{y\})$.

\section{Morphological geodesic erosion}

The morphological geodesic erosion is computed using the distance transform from $X^{c}$ in $X$, and by thresholding it, as defined below. 
Let $\varepsilon_{r}(X)$ be the eroded set (Serra, 1982) of $X$ by a sphere of radius $r \in \mathbb{N}$, defined by

$$
\varepsilon_{r}(X)=\left\{x \in X, D\left(x, X^{c}\right)>r\right\}
$$

with $D\left(x, X^{c}\right)$ the value of the distance transform to the complementary set of $X$, at point $x \in X$.

\section{Geodesic Distance Transform}

The geodesic distance transform (Rutovitz, 1968) is the equivalent of the distance transform in a non-Euclidean space. Geodesy could be seen as the restriction of the distance transform to a subset of the image (Lantuéjoul and Beucher, 1981).

Hereafter, $S$ is a subset of $X$. The binary geodesic distance transform $D_{G}(., S ; X)$ of each point $x \in X$, from the set $S$, restricted to the support $X$ is defined as

$$
D_{G}(x, S ; X)=\min _{\left\{x^{\prime} \in S\right\}} d_{X}\left(x, x^{\prime}\right)
$$

with

$$
d_{X}(x, y)=\inf _{\Gamma \in \gamma_{x, y} ; X} \int_{y}^{x} d s
$$

where $\gamma_{x, y ; X}$ is the set of all possible paths in $\mathbb{R}^{3}$ constrained by $X$, between $y \in X$, starting point, and $x \in X$, ending point. $\Gamma$ is one of these paths, and $s \in$ $[0, L(\Gamma)]$ its curvilinear abscissa, with $L(\Gamma)$ the length of the path $\Gamma$.

$D_{G}(S ; X)$ is the $3 \mathrm{D}$ geodesic distance map and, $\forall x \in X$, $D_{G}(x, S ; X)$ is the geodesic distance transform value at point $x$ from $S$, restricted to $X$. In the specific case of a singleton, $S=\{y\}, D_{G}(x, y ; X)$ stands for $D_{G}(x,\{y\} ; X) . D_{G}(x, y ; X)$ "is the greatest lower bound of the lengths of the $\operatorname{arcs}$ in $X$ ending at points $x$ and $y$, if such arcs exist, and $+\infty$ if not" (Lantuéjoul and Beucher, 1981). In this article, we use the convention $" 1 / \infty=0 "$, on which the definitions of the following descriptors are based.

\section{M-TORTUOSITY ESTIMATOR}

The notations are the same as used in Chaniot et al. (2019), where the M-tortuosity was formally defined. In a first step, the homotopic skeleton $S k$ (Saha et al., 2016) of the porous network $X$ is computed using the method of Lohou and Bertrand (2005). The starting points set, $S \subset S k \backslash\{c\}$, is defined by a stratified sampling method (Neyman, 1934); $N \in \mathbb{N}^{*} \backslash\{1,2\}$ points are sampled, $S=\left\{p_{n}\right\}_{n \in \llbracket 0 ; N-1 \rrbracket}$, with for all $n \in[[0 ; N-1]], p_{n} \in S k$.

\section{Morphological tortuosities}

The morphological tortuosity (Decker et al., 1998; Peyrega and Jeulin, 2013), normalization of the binary geodesic distance (Lantuéjoul and Beucher, 1981), between $p_{n} \in S$ and $p_{m} \in S, m \neq n$, denoted $\tau_{n, m}$, is defined as

$$
\tau_{n, m}=\frac{D_{G}\left(p_{m}, p_{n} ; S k\right)}{D\left(p_{m}, p_{n}\right)}
$$

with $D_{G}\left(p_{m}, p_{n} ; S k\right)$ the geodesic distance restricted to $S k$, and $D\left(p_{m}, p_{n}\right)$ the Euclidean distance, both between $p_{n}$, starting point, and $p_{m}$, ending point (Rosenfeld and Pfaltz, 1968; Borgefors, 1986).

\section{M-coefficients}

For $n \in\left[[0, N-1]\right.$, an estimated $M$-coefficient $\hat{C}_{n}$, attached to $p_{n}$, is defined using the harmonic mean of the morphological tortuosities.

$$
\begin{aligned}
\hat{C}_{n}^{-1} & =\frac{\sum_{m=0, m \neq n}^{N-1} \frac{1}{D_{G}\left(p_{m}, p_{n} ; S k\right)}}{\sum_{m=0, m \neq n}^{N-1} \frac{1}{D_{G}\left(p_{m}, p_{n} ; S k\right) \cdot \tau_{n, m}}} \\
\text { if, } \sum_{m=0, m \neq n}^{N-1} \frac{1}{D_{G}\left(p_{m}, p_{n} ; S k\right) \cdot \tau_{n, m}} & \neq 0
\end{aligned}
$$

The harmonic mean is defined by the reciprocal of the arithmetic mean of the reciprocals of the values, here the morphological tortuosities. The weighing, using geodesic distances (Berrocal et al., 2016), is justified as follows: the longer is the path, the more representative it is of the overall porous structure tortuosity.

\section{M-scalar}

The final scalar value, the $M$-scalar $\hat{\tau}_{M}$, is defined as

$$
\begin{aligned}
& \hat{\tau}_{M}=\frac{\sum_{n=0}^{N-1} \frac{1}{D\left(p_{n}, c\right)}}{\sum_{n=0}^{N-1} \frac{1}{D\left(p_{n}, c\right) \cdot \hat{C}_{n}^{-1}}} \\
& \text { if, } \sum_{n=0}^{N-1} \frac{1}{D\left(p_{n}, c\right) \cdot \hat{C}_{n}^{-1}} \neq 0
\end{aligned}
$$

The idea behind this second weighing is close to the previous one: the farther a point is from the center of mass of the network, the higher is the probability to get long paths in its corresponding $M$-coefficient value. 


\section{M-tortuosity specificities}

The $M$-tortuosity descriptor, $\hat{T}_{M}$, provides a single scalar value, $\hat{\tau}_{M}$, gathering topological information to characterize the overall microstructure of interest, as for porous media or for any phase of a multiphasic material. This is a scalable descriptor, i.e., information of various dimensions is available by means of 3D maps, histograms and scalar values. Moreover, as shown in Chaniot et al. (2019), this estimator is translation, rotation and homothety invariant, as well as stable when the pattern is periodical. The complexity is approximately in $O\left(N_{\mathbb{I}}\right)$, with $N_{\mathbb{I}}$ the number of discrete points of the image $\mathbb{I}$.

Thanks to the use of harmonic means, $\hat{T}_{M}$ can be applied to complex disconnected networks. To be more specific, the proposed descriptors handle disconnections and promote long geodesic paths. Therefore, in order to average the different view points from a random location, i.e., the different tortuosities associated with a specific source point in the definition of the M-coefficient (Eq. 7), and to respect the above constraints too, the harmonic mean seems to be an ideal candidate. This definition of the $M$ coefficient enables to handle disconnections between points belonging to distinct connected components of the microstructure. The second harmonic mean, in the definition of the $M$-scalar, allows to avoid the consideration of isolated points, by attributing to their $M$-coefficient a zero weight.

The M-tortuosity has been built so as to be directly computable on the microstructure of interest $X$. Nevertheless, when it is appropriate, the homotopic skeleton of the microstructure $S k$ can be used. We refer the reader to Chaniot et al. (2019), for the conditions under which the skeleton is chosen to be used, with an analysis of its impact on the results.

The situation "when at least two points are connected" is considered. If this is not the case, our descriptor is not suitable. In this context, the use of a Monte Carlo method is justified in Chaniot et al. (2019), as the specific conditions are fulfilled. First, the subset is bounded. Secondly, the descriptor is integrable and positive ; therefore, it belongs to $L^{1}$ space ; it also belongs to $L^{2}$ space of square integrable functions. According to Caflisch (1998), these conditions place our estimator and its variance, on a firm theoretical ground. Finally, the stabilisation of $N \sigma^{2}$ as a function of $N$ highlights the existence of the asymptotic domain, reached around $N$ equal to 50, with $N$ the number of random points and $\sigma^{2}$ the variance of the $M$-tortuosity values, the $M$-scalars, over realizations of a given stochastic model.

\section{M-TORTUOSITY-BY-ITERATIVE- EROSIONS ESTIMATOR}

\section{Definition}

Extension of $\hat{T}_{M}$, by using iterative erosions of $X$, allows to take into account of bottleneck effects by quantifying their impact over the morphological tortuosity, as illustrated in Fig. 2. $\hat{T}_{M, r}$ assesses the tortuosity of the accessible porous part for a given spherical probe size, $r$. This estimator is connected to the constrictivity concept (Petersen, 1958; Holzer et al., 2013; Neumann et al., 2019).
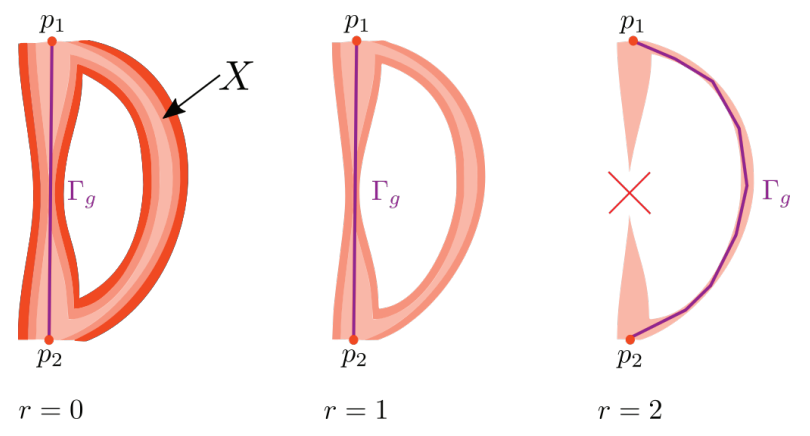

Fig. 2: Illustration of bottleneck effects on the morphological tortuosity between the two points $p_{1}$ and $p_{2}$, both belonging to $X$ (colored part). Each shade of orange represents a step. From left to right; at $r=0$ the geodesic path between $p_{1}$ and $p_{2}, \Gamma_{g}$, is straight, at $r=1$ the situation is unchanged, but at $r=2$ the bottleneck is closed, represented by a red cross, and $\Gamma_{g}$ is not straight anymore, the morphological tortuosity increases.

The M-tortuosity descriptor is applied on the eroded set of $\mathrm{X}, \varepsilon_{r}(X)$, by a sphere of radius $r$ (Eq. 3). $\hat{T}_{M, r}$ is defined for any integer $r$ by

$$
\hat{T}_{M, r}(X)=\hat{T}_{M}\left(\varepsilon_{r}(X)\right) .
$$

\section{M-tortuosity-by-iterative-erosions specificities}

The use of iterative morphological operations decreasing step by step the porous volume, has been already investigated, as in Vogel (2002); Wernert et al. (2010); Ohser et al. (2012).

In Vogel (2002), successive morphological openings are applied to the porous microstructure. The morphological opening consists of a morphological erosion followed by a morphological dilation of the network (Serra, 1982). The opening assesses the volume where the structuring element can be fully included inside the porous phase. Nevertheless, the disconnections are highlighted by the erosion, 
assessing the volume where the center of the structuring element can be located such that the structuring element is fully included inside the porous phase. Hereafter, only the morphological erosion is kept, as in Ohser et al. (2012), for computation time efficiency purposes.

Papers dealing with percolation (Jeulin and Moreaud, 2006) often consider a given propagation direction. Our descriptors are free from propagation directions thanks to the random sampling, and then do consider the percolation in a more stochastic manner. Percolation assesses the existence of paths between entries and exits. Usually, when images are considered, a face is defined as the entry and the opposite one as the exit. Here, the random points are the entries and the exits, which can be useful when arbitrary entries and exits are too artificial.

Despite these differences, the idea behind the proposed descriptors is similar. The tortuosity and the constrictivity, originally defined for simple pores, a sinuosidal cylinder with a constant radius and a straight cylinder with varying radii, respectively, are extended to the overall microstructure, as for the $M$ tortuosity-by-iterative-erosions.

These articles attest the interest of considering various particle sizes for topological characterization, allowing the analysis of transport properties using their connections with structural features, in particular in Wernert et al. (2010) where experimental investigations are carried out.

Global topological characterization of the overall structure is made possible thanks to these two descriptors, but local information is not clearly highlighted. The H-tortuosity aims to provide information about local features by quantifying topological variations as a function of the scale. First, the deterministic operator is defined, then estimators are proposed.

\section{H-TORTUOSITY}

\section{Peripheral subsets}

Let $\mathscr{P}_{x, d} \subset X$ defined by $\mathscr{P}_{x, d}=\left\{y_{x, d} \in\right.$ $\left.X ; D\left(y_{x, d}, x\right)=d, d \in \mathbb{R}^{*}\right\}$, with $D\left(y_{x, d}, x\right)$ the Euclidean distance between $x$ and $y_{x, d} . \mathscr{P}_{x, d}$ is a sphere of radius $d$ centered in $x$.

\section{Morphological tortuosities}

For each $x \in X \backslash\{c\}$, the morphological tortuosity between $x$ and $y_{x, d}$, named $\tau_{x, y_{x, d}}$, is defined as the ratio of the geodesic distance, length of the shortest path, to the Euclidean distance, length of the straight path, between these two points (Decker et al., 1998).

$$
\tau_{x, y_{x, d}}=\frac{D_{G}\left(y_{x, d}, x ; X\right)}{D\left(y_{x, d}, x\right)}
$$

with $D_{G}\left(y_{x, d}, x ; X\right)$ and $D\left(y_{x, d}, x\right)$, the geodesic distance constrained by $X$ and the Euclidean distance, respectively, both between $x$, the starting point, and $y_{x, d}$, the ending point.

\section{$H$-coefficients}

Given $x \in X \backslash\{c\}$, the $H$-coefficients associated with $x, C_{x}(d)$, with $d \in \mathbb{R} *$, are defined using the harmonic mean of the morphological tortuosities $\tau_{x, y_{x, d}}, y_{x, d} \in \mathscr{P}_{x, d}$, weighted by the inverse of the respective geodesic distance $D_{G}\left(y_{x, d}, x ; X\right)$.

$$
\begin{aligned}
C_{x}^{-1}(d) & =\frac{\int_{\mathscr{P}_{x, d}} \frac{1}{D_{G}\left(y_{x, d}, x ; X\right)} \mathrm{d} y_{x, d}}{\int_{\mathscr{P}_{x, d}} \frac{1}{D_{G}\left(y_{x, d}, x ; X\right) \cdot \tau_{x, y_{x, d}}} \mathrm{~d} y_{x, d}} \\
& \text { if, } \int_{\mathscr{P}_{x, d}} \frac{1}{D_{G}\left(y_{x, d}, x ; X\right) \cdot \tau_{x, y_{x, d}}} \mathrm{~d} y_{x, d} \neq 0
\end{aligned}
$$

This weighing has the advantage to keep the same weighing as for the M-tortuosity, and increases the discriminative power of the final descriptor, by lowering the influence of straight paths. The harmonic mean allows to handle disconnections, where the geodesic distance is equal to infinity. The denominator is equal to zero only if, for a given $d$, all the $y_{x, d}$ are disconnected to $x$. In this case, we impose $C_{x}(d)=0$.

\section{$H$-scalars}

The $H$-scalars, $\tau_{H}(d)$, are defined as the harmonic mean of $\left\{C_{x}^{-1}(d)\right\}_{x \in X \backslash\{c\}}$, weighted by the inverse of the respective Euclidean distance from $c, D(x, c)$.

$$
\begin{aligned}
\tau_{H}(d) & =\frac{\int_{X \backslash\{c\}} \frac{1}{D(x, c)} \mathrm{d} x}{\int_{X \backslash\{c\}} \frac{1}{D(x, c) \cdot C_{x}^{-1}(d)} \mathrm{d} x} \\
& \text { if, } \int_{X \backslash\{c\}} \frac{1}{D(x, c) \cdot C_{x}^{-1}(d)} \mathrm{d} x \neq 0
\end{aligned}
$$

The purpose of this second weighing is to have the same weighing as with the M-tortuosity. The integral of the denominator is equal to zero only if, for a given $d$, each point of $X \backslash\{c\}$ is disconnected from the others.

The deterministic definition of the H-tortuosity descriptor, $T_{H}$, is not suitable for large volumes 
processing, as in Chaniot et al. (2019). An estimator of the H-tortuosity, $\hat{T}_{H}$, based on Monte Carlo method (Caflisch, 1998), allows such processing. This will be done using a skeletonization step (Saha et al., 2016) and a point sampling approach. The next section introduces the successive steps giving rise to $\hat{T}_{H}$, the $\mathrm{H}$-tortuosity estimator.

\section{H-TORTUOSITY ESTIMATOR}

\section{Skeletonization (optional)}

The homotopic skeleton $S k$ of the porous network $X$ is computed, and then characterized instead of $X$, for the same reasons than the ones presented in Chaniot et al. (2019). Nevertheless, the H-tortuosity can be computed on $X$, without the homotopic skeleton computation. The skeletonization introduces a desired bias, this has been shown partly in Chaniot et al. (2019). The homotopic skeleton seems to be a good candidate to decrease the computation time, and increase the discriminative power (Chaniot et al., 2019). A direct characterization of the interconnected complex porous network is impaired by the high number of non relevant straight paths. In other words, using the skeleton allows to decrease the number of paths which do not characterize the global topology of the overall microstructure.

In this paper, the thinning method of Lohou and Bertrand (2005), using the notion of P-simple point, is chosen. The obtained skeleton, named $S k$, is the smallest homotopic subset of $X$.

\section{Sampling}

The sampling method has a central role in the descriptor definition. It has to be chosen very carefuly, because the analysis of the results depends on it. In this paper, a stratified sampling is chosen (Neyman, 1934), and compared to the 1D-sampling method. Both sampling methods are first defined and then illustrated in Fig. 3, as in Chaniot et al. (2019).

\section{D-sampling:}

This sampling strategy corresponds to a uniform sampling in the pore phase skeleton. $S k$ can be rewritten as $S k=\left\{x_{i}\right\}_{i \in \llbracket 0,|S k|-1 \rrbracket}$, which can be seen as an indexing of $S k$, with $|S k|$ its number of elements. Uniform distribution $U([0,|S k|-1])$ allows to randomly draw $N$ distinct points in $S k \backslash\{c\}$, defining $S$.

\section{Stratified sampling:}

This sampling strategy (Baddeley and Jensen, 2005) corresponds to a uniform density of sampled points in $S k$ (Neyman, 1934). Let $\left\{W_{i}\right\}_{i \in[0, K-1 \rrbracket}$ be a set of $K$ sub-images of $\mathbb{I}$ with $K=k^{3}, k \in \mathbb{N}$ and 3 corresponds to the dimension of the space. If $S k \cap$ $W_{i} \neq \emptyset$, as before, one point is uniformly drawn in $S k \cap W_{i} \backslash\{c\} . N$ points are sampled in $S k \backslash\{c\}$, with $N \leq K$, defining $S$.

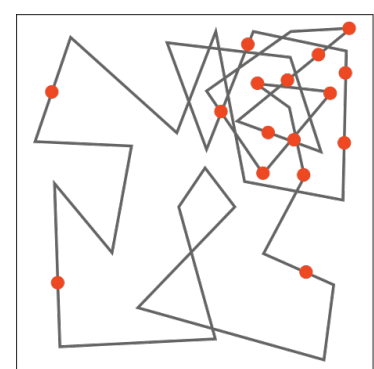

(a)

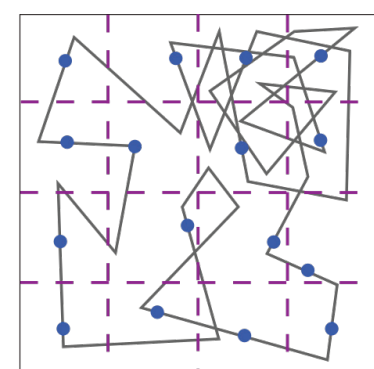

(b)
Fig. 3: Examples of skeleton $S k$, represented by the broken lines, with the (a) 1D-sampling and (b) stratified sampling methods. $S$ is the set of orange or blue dots (pictures from Chaniot et al. (2019)).

\section{Quantitative comparison:}

In Chaniot et al. (2019), the stabilisation of $N \sigma^{2}$, with $N$ the number of random points and $\sigma^{2}$ the variance of the $M$-tortuosity value over a certain number of realizations, is given with results over 100 images of size $200^{3}$, of a Boolean model of spheres of radius $R=3$ and volume fraction $V_{v}=0.7$. This has been done considering the $1 \mathrm{D}$-sampling method.

The stratified sampling method has a specificity; the requested number of random points, given by the user, is not always equal to the final number of points defining $S$. This is why in the results presented in Tab. 1 some boxes are blank.

The M-tortuosity is computed over the same set of realizations of the same model, but considering the stratified sampling method. For comparison purposes, $N \sigma^{2}$ is computed for various values of $N$, not equal for both methods, but close enough to allow a meaningful comparison.

Whatever the value of $N$, the variance $\sigma^{2}$ is lower with the stratified sampling method. Moreover, this method gets faster convergence (Baddeley and Jensen, 2005). Consequently, the stratified sampling method seems well adapted, considering the nature of the images, in order to characterize the overall microstructure. For all these reasons, the stratified sampling is chosen for the results below. 


\begin{tabular}{|c|c|c|}
\hline$N$ & $N \sigma^{2}(1 \mathrm{D})$ & $N \sigma^{2}$ (stratified) \\
\hline 5 & 0.0213 & \\
\hline 8 & & 0.0037 \\
\hline 10 & 0.0075 & \\
\hline 60 & 0.0076 & \\
\hline 64 & & 0.0037 \\
\hline 70 & 0.0103 & \\
\hline 100 & 0.0081 & 0.0027 \\
\hline
\end{tabular}

Table 1: $N \sigma^{2}$ as a function of $N$ for the both sampling methods: 1D and stratified.

\section{Discrete peripheral subsets}

For a given $p_{n} \in S$, let $P_{n, d} \subset S k$ defined by $P_{n, d}=\left\{s_{n, d, m} \in S k ; d<D\left(s_{n, d, m}, p_{n}\right) \leq d+\right.$ $\left.1, d \in \mathbb{N}^{*}\right\}_{m \in \llbracket 0, M-1 \rrbracket}$, with $D\left(s_{n, d, m}, p_{n}\right)$ the Euclidean distance between $p_{n}$ and $s_{n, d, m}$, and $M$ the number of points in $P_{n, d}$, for $n$ and $d$ given (Fig. 4 (a)).

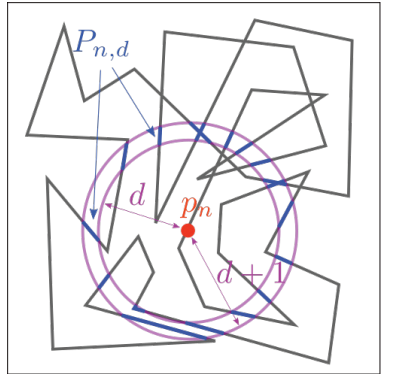

(a)

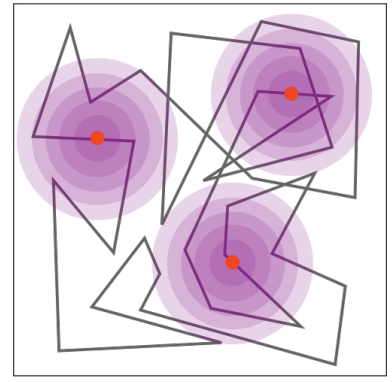

(b)
Fig. 4: Examples of skeleton $S k$, represented by the broken lines, with representations of (a) the peripheral subset $P_{n, d}$ for $p_{n} \in S$ and $d$ given, and (b) the local probing of the microstructure around each point of $S$.

\section{Morphological tortuosities}

Given $S$, the morphological tortuosity (Decker et $a l ., 1998)$ between $p_{n} \in S$ and $s_{n, d, m} \in P_{n, d}$ named $\tau_{n, d, m}$, is defined as

$$
\tau_{n, d, m}=\frac{D_{G}\left(s_{n, d, m}, p_{n} ; S k\right)}{D\left(s_{n, d, m}, p_{n}\right)}
$$

with $D_{G}\left(s_{n, d, m}, p_{n} ; S k\right)$ and $D\left(s_{n, d, m}, p_{n}\right)$ the geodesic distance and the Euclidean distance, respectively, between $p_{n}$, starting point, and $s_{n, d, m}$, ending point.

\section{$H$-coefficients estimator}

Given $n \in[[0, N-1]]$ and $d \in \mathbb{R}^{*}$, the estimated $H$-coefficients $\hat{C}_{n}(d)$ are defined as

$$
\begin{aligned}
\hat{C}_{n}^{-1}(d) & =\frac{\sum_{m=0}^{M-1} \frac{1}{D_{G}\left(s_{n, d, m}, p_{n} ; S k\right)}}{\sum_{m=0}^{M-1} \frac{1}{D_{G}\left(s_{n, d, m}, p_{n} ; S k\right) \cdot \tau_{n, d, m}}} \\
\text { if, } \sum_{m=0}^{N-1} \frac{1}{D_{G}\left(s_{n, d, m}, p_{n} ; S k\right) \cdot \tau_{n, d, m}} & =0
\end{aligned}
$$

The H-coefficients assesses the neighboring tortuosities as a function of the point $p_{n}$ and the distance $d$. For a given location, $\hat{C}_{n}(d)$ probes the peripheral porous network, as shown in Fig. 4 (b).

\section{$H$-scalars estimator}

The estimated $H$-scalars, attached to $d$, are

$$
\begin{aligned}
\hat{\tau}_{H}(d) & =\frac{\sum_{n=0}^{N-1} \frac{1}{D\left(p_{n}, c\right)}}{\sum_{n=0}^{N-1} \frac{1}{D\left(p_{n}, c\right) \cdot \hat{C}_{n}^{-1}(d)}} \\
& \text { if, } \sum_{n=0}^{N-1} \frac{1}{D\left(p_{n}, c\right) \cdot \hat{C}_{n}^{-1}(d)} \neq 0 .
\end{aligned}
$$

$\hat{\tau}_{H}(d)$ allows to assess the average tortuosity at a given distance $d$ for a random point. Heterogeneity is quantified by its impact on the average variations of tortuosity as a function of the scale, i.e. Euclidean distances, for a scale range limited by the volume size. The H-tortuosity $\hat{T}_{H}$ accounts for the scale heterogeneity, the way morphological tortuosity varies according to the measurement scale (d).

The approximate complexity of the H-tortuosity algorithm, as the M-tortuosity algorithm, is $O\left(N_{\mathbb{I}}\right)$.

\section{H-TORTUOSITY-BY-ITERATIVE- EROSIONS ESTIMATOR}

Morphological erosion is used for defining the H-tortuosity-by-iterative-erosions estimator, $\hat{T}_{H, r}$, assessing the average variations of tortuosity according to the scale, but for a spherical percolating particle of a given size $r$. The idea behind this new descriptor is to consider only the accessible porous part for a given probe size. $\hat{T}_{H, r}$ is defined by

$$
\hat{T}_{H, r}(X)=\hat{T}_{H}\left(\varepsilon_{r}(X)\right) .
$$

As for the M-tortuosity-by-iterative-erosions, $\hat{T}_{H, r}$ allows to take into account local narrowness of $X$, and especially bottleneck effects. The H-tortuosityby-iterative-erosions gathers the concepts of tortuosity and constrictivity (Petersen, 1958; Holzer et al., 2013; Neumann et al., 2019), to quantify heterogeneity. 
Moreover, as for the M-tortuosity, the M-tortuosity-byiterative-erosions and the $H$-tortuosity, the percolation concept is considered in a non-conventional way; existence of paths between random points.

Similarly to the previously cited descriptors, $\hat{T}_{H, r}$ can be applied to the microstructure or to its skeleton. Contrary to Eq. 16, we choose, still for the computation time efficiency, to use a unique random points set $S$, which means there is no generation of a new set $S$ at each value of $r$. Moreover, instead of computing the morphological erosion at each iteration, the skeleton $S k$ is used and valued using the distance map $D$; to each point of the skeleton is attributed the distance value at the exact same location. Consequently, the erosion is perceived as a decrementation of the values of $S k$. These choices have a significant impact on the results. The same connections are considered during the computations, as the set of random points is generated once. The skeleton allows to highlight, strongly, the impact of bottlenecks: the minimal path between two points in a bottlenecked pore remaining the same, the tortuosity remains constant, until this specific path is disconnected. This is not the case without the skeletonization.

\section{NUMERICAL METHODS}

The $H$-tortuosity and the $H$-tortuosity-by-iterativeerosions are numerical descriptors. The algorithms, or the computational methods, are defined below using pseudo-codes. Three main aspects of our methods are presented too: connectivity, being a central issue of any topological analysis, the geodesic distance transform on which our descriptors are based, where all the topological information is, the third one being sampling, with its impact on the meaning of the results.

\section{$H$-tortuosity}

As for the M-tortuosity, the H-tortuosity is a scalable descriptor; topological information of different dimensions can be extracted from the algorithm. The numerical definition below allows to highlight this specific asset of our descriptors.

The very first computations of the H-tortuosity algorithm are identical to the M-tortuosity algorithm. The H-tortuosity has a certain sampling, as its $N$ random points are sampled in $S k$, defining the set $S=\left\{p_{i}\right\}_{i \in \llbracket 0, N-1 \rrbracket}$. The distance map and the geodesic distance map are computed for each point of $S, D\left(., p_{n}\right)$ and $D_{G}\left(., p_{n} ; S k\right)$, respectively. A morphological tortuosity map, $\mathbb{T}\left(., p_{n} ; S k\right)$, is deduced from these two maps, for each source point.

In the following, $D_{G}\left(., p_{n} ; S k\right), D\left(., p_{n}\right)$ and $\mathbb{T}\left(., p_{n} ; S k\right)$ will be written $D_{G}\left(p_{n}\right), D\left(p_{n}\right)$ and $\mathbb{T}\left(p_{n}\right)$ for brevity. We propose to define the $3 D$ map of mean relative tortuosities, named $\overline{\mathbb{T}}(., S ; S k)$. For each location $x \in$ $S k$, we compute the average over the $N$ morphological tortuosity maps $\mathbb{T}\left(p_{n}\right)$, but considering only the finite values, which is the origin of the term relative. Indeed, infinite values account for disconnections, which is not our objective, at least for this specific map.

Subsequently, each $\mathbb{T}\left(p_{n}\right)$ is probed starting from $p_{n}$ considering $d \in\left[\left[1, D_{\max }-1\right]\right]$ and a given step, here chosen equal to $1 . D_{\max }$ is the maximal probing distance imposed by the user. The $H$-coefficients are defined for each pair $\left(p_{n}, d\right)$ (Eq. 13 and 14). Here again, the $H$-coefficients could be used as descriptors, more specifically of peripheral tortuosities.

Finally, the $H$-scalars $\hat{\tau}_{H}(d)$ are computed using Eq. 15.

The H-tortuosity descriptor is defined by this computation process of which a detailed description is given by the following pseudo-code (Algo. 1).

Algorithm 1: H-tortuosity

Result: $H$-scalars $\hat{\tau}_{H}(d)$

Skeleton computation $S k$; Generation of $S=\left\{p_{i}\right\}_{i \in \llbracket 0, N-1 \rrbracket}$;

for $n \in[[0, N-1]]$ do

Computation of:

Geodesic distance map $D_{G}\left(p_{n}\right)$;

Euclidean distance map $D\left(p_{n}\right)$;

Morphological tortuosity map $\mathbb{T}\left(p_{n}\right)$;

Computation of:

$3 D$ map of mean relative tortuosities

$\overline{\mathbb{T}}(., S ; S k)$;

for $\left.d \in\left[1, D_{\max }\right]\right] \mathbf{d o}$

$H$-coefficient computation:

$$
\hat{C}_{n}^{-1}(d)=\frac{\sum_{m=0}^{M-1} \frac{1}{D_{G}\left(s_{n, d, m}, p_{n} ; S k\right)}}{\sum_{m=0}^{M-1} \frac{1}{D_{G}\left(s_{n, d, m}, p_{n} ; S k\right) \cdot \tau_{n, d, m}}}
$$$$
\text { end }
$$

end

H-scalars computation:

$$
\hat{\tau}_{H}(d)=\frac{\sum_{n=0}^{N-1} \frac{1}{D\left(p_{n}, c\right)}}{\sum_{n=0}^{N-1} \frac{1}{D\left(p_{n}, c\right) \cdot \hat{C}_{n}^{-1}(d)}} ;
$$




\section{H-tortuosity-by-iterative-erosions}

The computational method of $\hat{T}_{H, r}$, as said above, is based on iterative morphological erosions, simulated by the decrementation of the valued skeleton. $S k$ is decremented step by step, with $\hat{T}_{H}$ applied on $S k$ at each step, in other words for each considered value of $r . \hat{T}_{H}$ is suitable to allow the use of a unique pregenerated random points set.

In the following pseudo-code (Algo. 2), $D$ stands for the distance map to the complementary set $D\left(., X^{c}\right)$ (Eq. 1), for all $x \in X$, for brevity.

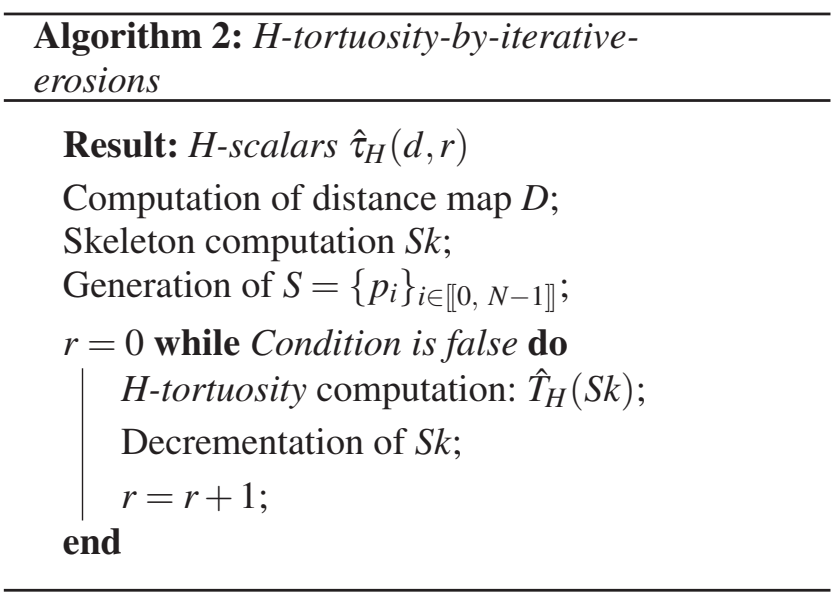

The stopping condition is arbitrary, but can be defined using the number of connected points in $S$, which could be used for promoting the consideration of percolation theory in the definition of the $\mathrm{H}$ tortuosity-by-iterative-erosions.

In the definitions of the H-tortuosity curve and the H-tortuosity-by-iterative-erosions curves, the heterogeneity is seen through the porosity. The heterogeneity of the morphological tortuosity according to the scale, as defined in this article, can be assessed by computing, for a given $d$, the standard deviation of all the morphological tortuosities $\tau_{n, d, m}$, for all $n$ and $m$. Heterogeneity is then seen through morphological tortuosity.

\section{Connectivity}

3D connectivity considers either 6,18 or 26 neighbor voxels, when a voxel's neighbor is defined, respectively, as a voxel sharing either a face, at least an edge or at least a vertex only. The considered connectivity degree has a non-negligible impact on digital image processing, especially for topological computation methods.

Global connectivity is defined by the choice of the foreground and background neighborhoods. In this paper, we take the most usual choice: $N_{26}-$ neighborhood for the foreground and $N_{6}$-neighborhood for the background.

\section{Geodesic Distance Transform}

Geodesic distance transforms are a very powerful tool for connectivity issues (Lantuéjoul and Beucher, 1981), and are at the very basis of our descriptors. In this article, only the binary geodesic distance transform is considered.

In Eq. 7, harmonic mean is used for turning disconnections into zero contributions. Indeed, the geodesic distance of two nonconnected points of $X$ is infinite. Consequently, using arithmetic mean, only one disconnection would cover up all the rest of the topological information. Using the inverse of the geodesic distance annihilates the contribution of disconnected paths; nonconnected points will not interfere in the computation. Moreover, considering the harmonic mean, this weighing allows to highlight the long geodesic paths.

The geodesic distance map $D_{G}\left(p_{n}\right)$ is computed using the raster scanning algorithm. A similar algorithm can be found in Toivanen (1996), for grayscale images. Basically, raster scans of opposite directions are used for propagating local distances named weights, until idempotence or stabilization, as shown in Fig. 5.

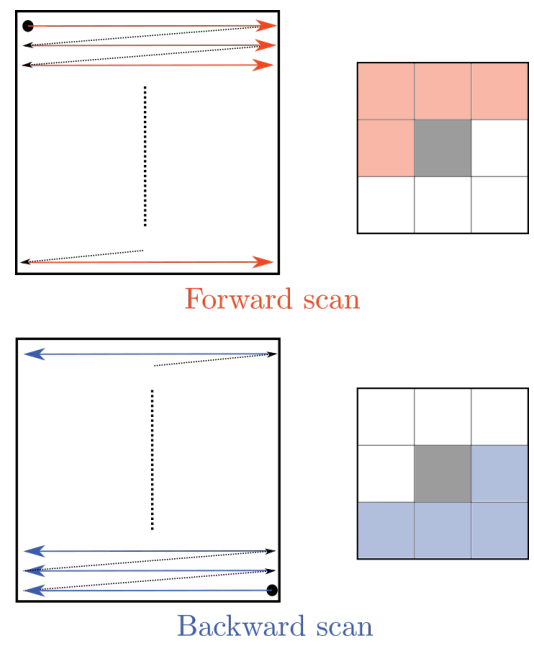

Fig. 5: The two raster scans of opposite directions: forward scan and backward scan, used in the raster scanning algorithm for the computation of the geodesic distance map.

The $N_{26}$-neighborhood for the foreground imposes three weights $\left(w_{1}, w_{2}, w_{3}\right)$ for direct, 2D-diagonal and 3D-diagonal neighbors, respectively. Hereafter, Euclidean weights are considered: $\left(w_{1}, w_{2}, w_{3}\right)=$ $(1, \sqrt{2}, \sqrt{3})$.

\section{Sampling}

The choice of the sampling method is totally correlated with the results meaning, and then their analysis. Whatever the chosen sampling strategy, 
to avoid boundary issues with the H-tortuosity computation, a sampling area is imposed, as shown in Fig. 6.

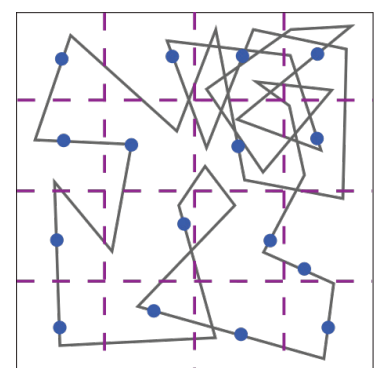

(a)

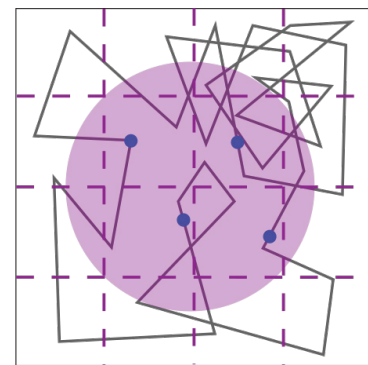

(b)
Fig. 6: Examples of skeleton $S k$, represented by the broken lines, with (a) the original set of sampling points computed by the stratified sampling method and (b) the final set $S$ considering the sampling area, the purple disk.

Let $(W, H, Z) \in \mathbb{N} *^{3}$, define the size of the image. Let $m_{W, H, Z}$ be the integer part of the half of the minimal value of $(W, H, Z)$. Considering the maximal value of $d, D_{\max }$, given by the user, a sphere centered at the image center with radius $m_{W, H, Z}-D_{\max }-1$ defines the sampling area. Consequently, for any point $p_{n} \in S$ and any value of $d$, the sphere centered at $p_{n}$ and of radius $d+1$, is totally included into the image.

Concerning the sampling method, as said above, the stratified sampling method is chosen due to its faster convergence and to its lower variance for similar values of $N$ (Tab. 1). Moreover, stratified sampling seems to be suitable to global structure characterization (Baddeley and Jensen, 2005).

Nevertheless, with this method, the user gives a target number of random points $N_{T}$ as parameter. The image is then divided into $K$ subimages, here cubes, but $K$ is contrained as $K=k^{3}, k \in \mathbb{N}$. Finally, the value of $N$ is only an approximation of $N_{T}$, equal to the number of non-empty subimages. Hereafter, we impose $K>N_{T}$, such that even in the case of empty cubes, $N$ is still around $N_{T}$. Obviously, this is no longer reliable in the case of high volume fraction of solid, leading to numerous empty subimages, which is not considered here. The convergence of $N \sigma^{2}$, studied in Chaniot et al. (2019) for the 1D-sampling, is an experimental proof of the method's stability. We chose $N$ around 64, based on the work of Chaniot et al. (2019).

\section{RESULTS AND DISCUSSION}

The usefulness of the H-tortuosity and the H-tortuosity-by-iterative-erosions is highlighted by applying them to Cox multi-scale Boolean models. A brief description of such models is proposed, followed by two specific cases with the purpose of attesting the importance of the complementarity of the M-tortuosity and the $\mathrm{H}$-tortuosity. Finally, the $\mathrm{H}$-tortuosity behavior is validated by showing its sensitivity to geometric heterogeneity. All these applications testify of its discriminative power, which increases by considering bottleneck effects using the $H$-tortuosity-by-iterativeerosions.

\section{BOOLEAN MODELS AND COX MULTI- SCALE BOOLEAN MODELS}

\section{Boolean models}

Boolean models (Matheron, 1975; Serra, 1982) are based on a Poisson Point process (Kingman, 1993). The number of points to be placed is a random variable, following a Poisson distribution. Random primary grains (overlapping allowed) are located at Poisson points.

For more details about Boolean models, please refer to Matheron (1975); Serra (1982); Chiu et al. (2013).

\section{Cox multi-scale Boolean models}

A multi-scale microstructure can be modelized by using Cox multi-scale Boolean models (Jeulin, 1996; Jean et al., 2011; Moreaud et al., 2018; Bortolussi et al., 2018). This process is defined by intersections and unions between objects and points, generated by several Poisson point processes, named Cox point processes (Jeulin, 1997; 2012). This way to model multi-scale complex microstructures gives more realistic results than intersections and unions of several Boolean models (Savary et al., 1999; Moreaud et al., 2018), in particular no grains are cut.

In this paper, the focus is mostly on two-scale models defined by three volume fractions: $V_{v, I N C}$, the volume fraction of inclusion areas (defining aggregates), $V_{v}$, the volume fraction of grains inside inclusion areas, and $V_{v, \text { OUT }}$, the volume fraction of grains outside of inclusion areas. Fig. 7 shows examples of two realizations of two distinct Cox multi-scale Boolean models using spheres (Fig. 7(a)) and platelets (Wang et al., 2015), i.e. rectangular cuboid (Fig. 7(b)).

Results on Boolean models and on Cox multiscale Boolean models are presented in the following. More specifically, we focus our attention on the complementary set of the set of primary grains. Hereafter, as Cox multi-scale Boolean models are considered, for a model, several realizations, i.e. images, are generated and the H-tortuosity is computed on each of them. The results we present, are an average 
over all the realizations. In order to avoid complicated notations, $\hat{\tau}_{H}(d)$, usually used for the H-tortuosity curve, stands for the average curve of all the $H$ tortuosity curves, obtained for each realization. As in Chaniot et al. (2019), the descriptors proposed in this article can be applied on any microstructure.

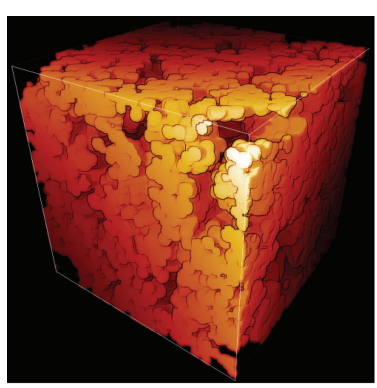

(a)

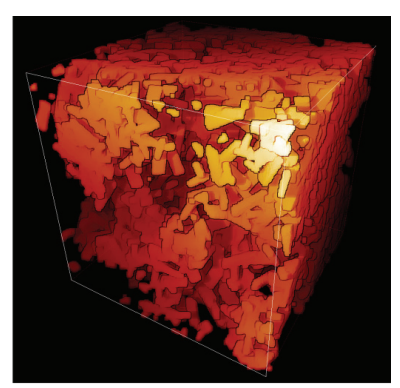

(b)
Fig. 7: Two realizations of two distinct Cox multiscale Boolean models of spheres (a) and platelets (b) (Moreaud et al., 2018). Volumes generated and rendered using "plug'im!" (2018).

The same set of starting points $S$ is used for the $M$ tortuosity, the M-tortuosity-by-iterative-erosions, the $H$-tortuosity and the H-tortuosity-by-iterative-erosions computations. This means that the sampling area, originally imposed only for the $\mathrm{H}$-tortuosity and the $\mathrm{H}$ tortuosity-by-iterative-erosions, is also used for the $M$ tortuosity and the M-tortuosity-by-iterative-erosions.

The stopping condition for the M-tortuosity-byiterative-erosions is "when all realizations are disconnected", which means that for each realization, each point of $S$ is isolated from the others. This is the least restrictive condition. In practice, we stop the display of curves when at least one realization is disconnected. The stopping condition for the $\mathrm{H}$ tortuosity-by-iterative-erosions is identical to the one used for the M-tortuosity-by-iterative-erosions. In practice, we stop the display of curves for an arbitrary small value of $r$, for a better visual analysis.

Finally, the confidence level is at $95 \%$; therefore the uncertainty is defined as twice the standard deviation over the $N$ realizations, divided by the square root of $N$.

\section{FROM M-TORTUOSITY TO H-TORTUOSITY}

The M-tortuosity fits to characterize global features of microstructures while the $\mathrm{H}$-tortuosity highlights local characteristics. Two cases are considered to show the complementarity between these two numerical descriptors.

\section{Boolean models of spheres with varying volume fraction}

First, Boolean models of spheres of radius $R=10$, with varying volume fraction of solid phase $V_{v}$, are generated. The 20 realizations, i.e. the images, are of size $400^{3}$, and are defined by:

1. $V_{v}=0.3$,

2. $V_{v}=0.4$,

3. $V_{v}=0.5$,

4. $V_{v}=0.6$,

5. $V_{v}=0.7$.

Fig. 8 shows two realizations of two distinct models, (1) and (5).

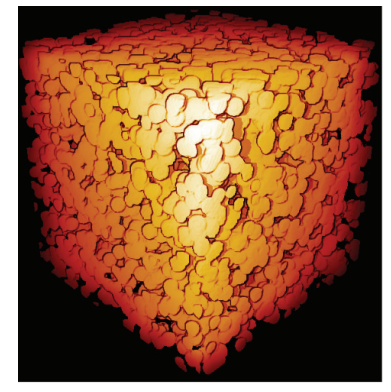

(1)

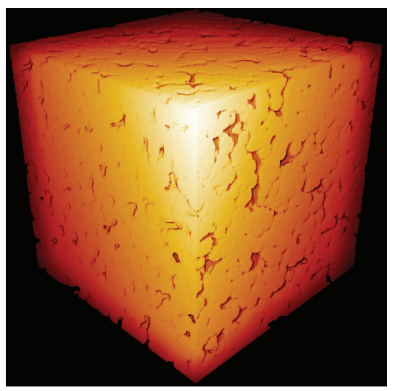

(5)
Fig. 8: Two realizations of two distinct Boolean models of spheres of radius $R=10$, (1) $V_{v}=0.3$ and (5) $V_{v}=0.7$. Volumes generated and rendered using "plug'im!" (2018).

$V_{v}$ is a global feature, consequently the $M$ tortuosity seems to be adapted to characterize and discriminate such models. Fig. 9 shows the $M$ tortuosity values (Fig. 9(a)), i.e. the $M$-scalars, and the M-tortuosity-by-iterative-erosions values (Fig. 9(b)), for each model.

For this case, the M-tortuosity is sufficient to discriminate the models, and the M-tortuosity-byiterative-erosions enhances the discriminative power, by considering bottleneck effects.

\section{Boolean models of spherocylinders with varying anisotropy}

The second case considers Boolean models of spherocylinders with varying anisotropy. A spherocylinder (Fig. 10) is a cylinder with two hemispherical caps at each end, thus defined by two parameters: $L$ the length of the cylinder and $R$ the radius of the hemispheres. The anisotropy $A$ of spherocylinders is defined as the ratio between their length, $L+2 R$, and their width, $2 R$. 


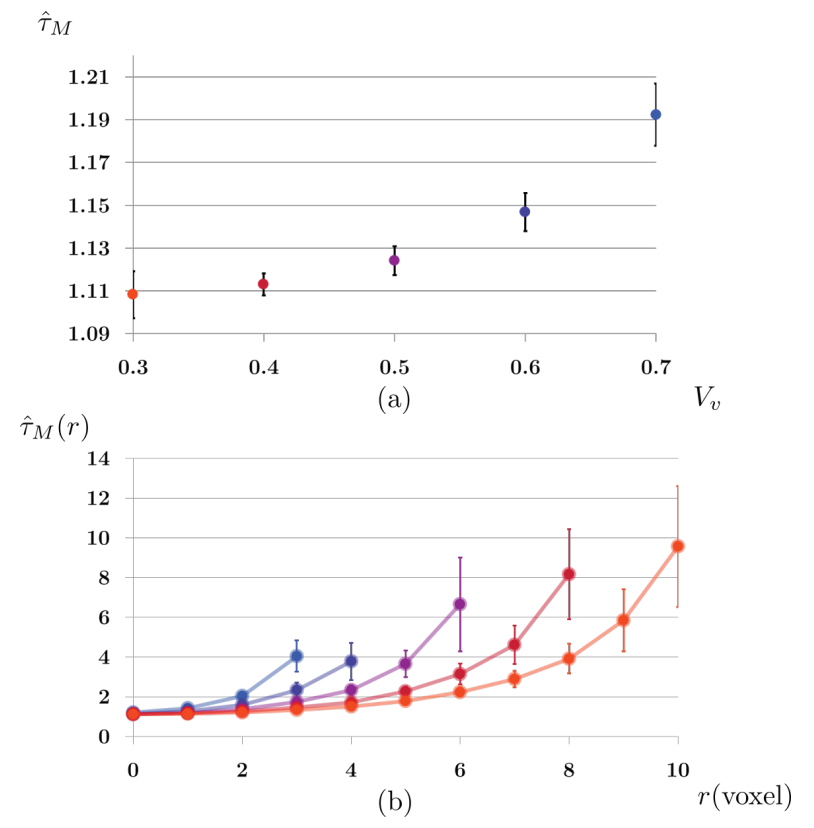

Fig. 9: (a) $\hat{\tau}_{M}$, the M-tortuosity value, as a function of $V_{v}$, the solid volume fraction of Boolean models of spheres of radius $R=10$, and (b) $M$-tortuosity-byiterative-erosions, $\hat{\tau}_{M}(r)$, for each model defined by $V_{v}$. Confidence intervals, with confidence level at $95 \%$, are represented by vertical bars.
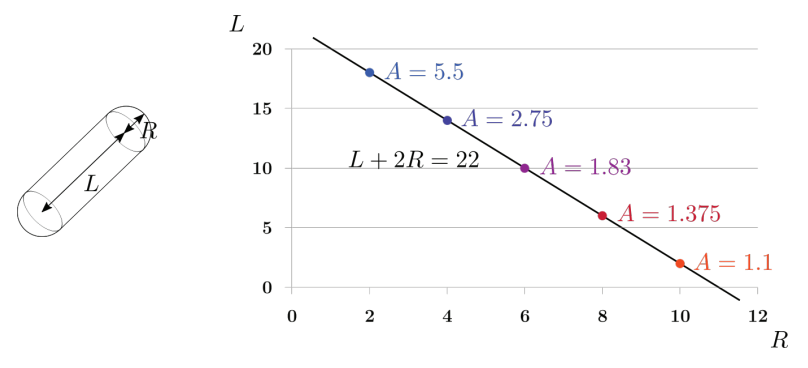

Fig. 10: Representations of the spherocylinder (left), defined by $L$ and $R$, and of the five models (right) with fixed morphological diameter $L+2 R$, defined by the spherocylinder's anisotropy $A$.

Five models are considered with various anisotropy and fixed morphological diameter $L+2 R$, as shown in Fig. 10. The volume of the spherocylinder $V$ is indicated too. They are defined by:

1. $A=1.1, L=2, R=10$ and $V \simeq 4817$,

2. $A=1.375, L=6, R=8$ and $V \simeq 3351$,

3. $A=1.83, L=10, R=6$ and $V \simeq 2036$,

4. $A=2.75, L=14, R=4$ and $V \simeq 972$,

5. $A=5.5, L=18, R=2$ and $V \simeq 260$.

20 realizations of size $400^{3}$ and $V_{v}$ equal to 0.5 , are generated for each model, i.e. each value of $A$. Fig. 11 shows two realizations of two distinct models, (1) and (3).

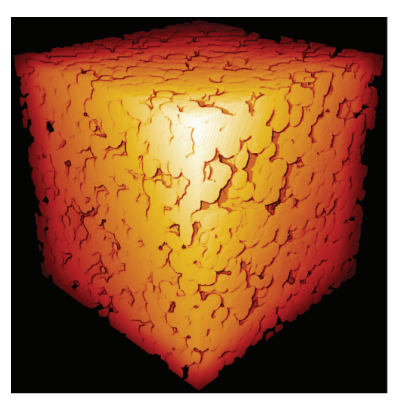

(1)

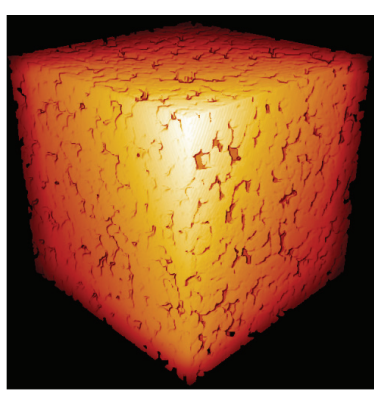

(3)
Fig. 11: Two realizations of two distinct Boolean models of spherocylinders, (1) $A=1.1$ and (3) $A=1.83$. Volumes generated and rendered using "plug'im!" (2018).

Anisotropy of grains is a local feature of such microstructures. Consequently, we can assume that the M-tortuosity fails to discriminate between the five models. Fig. 12(a) shows this limit.

In contrast, the $H$-tortuosity fits this specific case (Fig. 12(b-c)). Moreover, the local aspect of the grains anisotropy is highlighted. Indeed, Fig. 12(c) is an enlargment of Fig. 12(b), over small distances $d$, which is the key interval to discriminate models.

\section{H-TORTUOSITY FOR HETEROGENEITY CHARACTERIZATION}

The H-tortuosity is sensitive to local topological variations. Consequently, it fits for heterogeneity quantification, which is the origin of its name. To promote the H-tortuosity sensitivity to geometric heterogeneity, Cox multi-scale Boolean models of platelets are used (Wang et al., 2015).

The platelets, i.e. rectangular cuboids, are defined by three parameters: length $l$, width $L$ and height $H$. Hereafter, these parameters are fixed to $l=L=13$ and $H=3$.

In this specific case of Cox multi-scale Boolean models, geometric heterogeneity $\eta$ can be defined as

$$
\eta=\frac{V_{v}}{V_{v, \text { OUT }}} .
$$

We propose another parameter $D_{\eta}$ to enhance the geometric heterogeneity quantification. $D_{\eta}$ is defined as

$$
D_{\eta}=V_{v}-V_{v, \text { OUT }} .
$$

Two distinct cases are considered. The first one is composed of three Cox multi-scale Boolean models with $V_{v, O U T}, V_{v, I N C}$ and $R_{I N C}$ fixed, equal to $0.2,0.5$ and 20 , respectively. The different models are then defined by: 


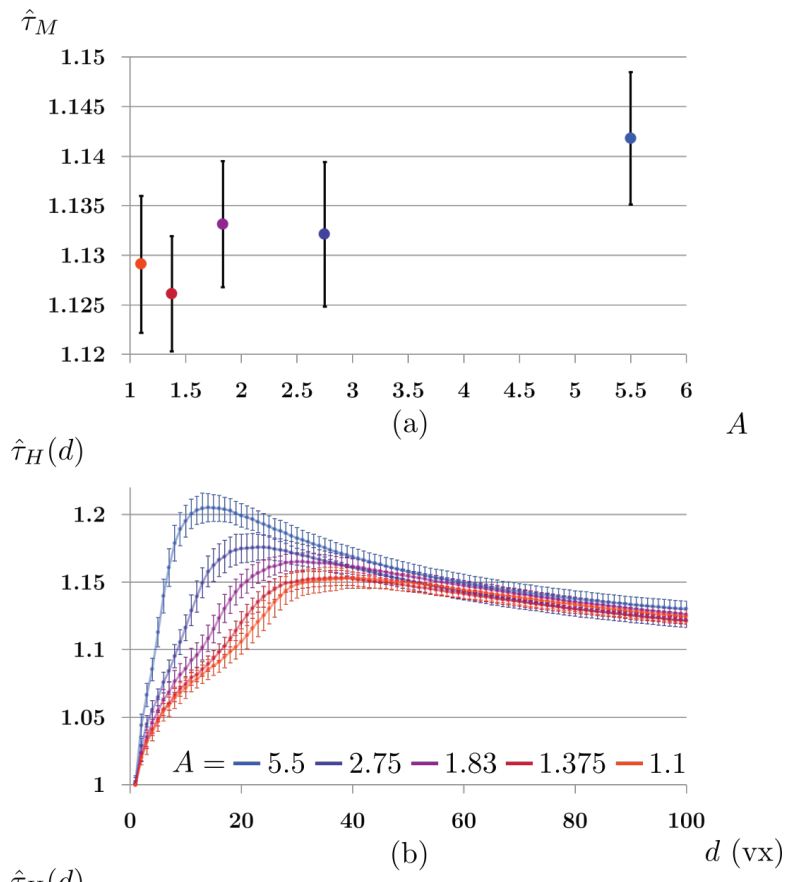

$\hat{\tau}_{H}(d)$

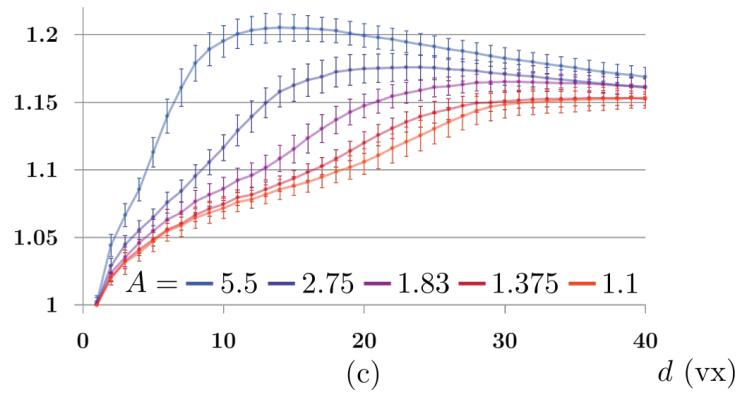

Fig. 12: (a) M-tortuosity value, $\hat{\tau}_{M}$, (b) H-tortuosity curve, $\hat{\tau}_{H}(d)$, of each models defined by the anisotropy $A$, and (c) zoom of (b) over small distances $d$. Confidence intervals, with confidence level at $95 \%$, are represented by vertical bars.

1. $V_{v}=0.2$,

2. $V_{v}=0.5$,

3. $V_{v}=0.8$.

The first model is a Boolean model, homogeneous, defined by $\eta=1$ and $D_{\eta}=0$. The two other models are defined by $\eta=2.5$ and $D_{\eta}=0.3$, and $\eta=4$ and $D_{\eta}=0.6$. Fig. 13 shows two realizations of two distinct models, (1) and (3). The indices are the same as the ones used in Fig. 14.

As expected, in Fig. 14(a-b) the higher the imposed geometric heterogeneity, the higher the morphological tortuosity, whatever the distance. This is mostly due to the fact that with Cox multi-scale Boolean models, the first scale, aggregation scale, prevails over the grain scale. Moreover, for each model, tortuosity reaches a maximum, at a certain value $d$, displaying, further on, a kind of stabilization. In this case, the maximal tortuosity and its corresponding abscissa discriminate the three models. The stabilization value, corresponding to the asymptotic tortuosity, could probably discriminate the models, but the maximal distance is not long enough in the present simulation to highlight this point. Finally, the analysis of these results highlights the local and global influence of geometric heterogeneity over topology variations.

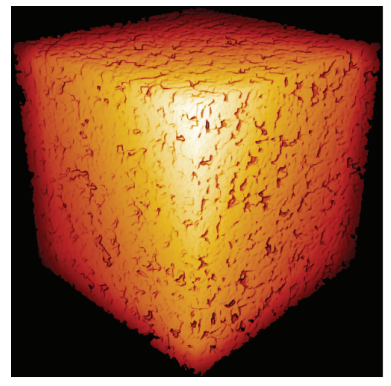

(1)

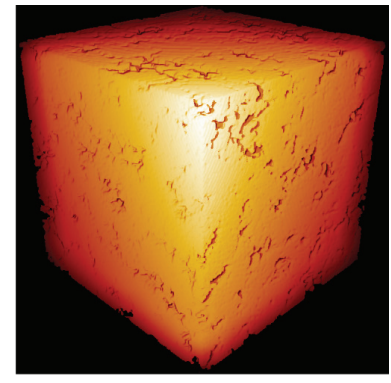

(3)
Fig. 13: Two realizations of two distinct Cox multiscale Boolean models of platelets $(l=L=13$ and $H=$ 3), (1) $V_{v}=0.2$ and (3) $V_{v}=0.8$. Volumes generated and rendered using "plug'im!" (2018).

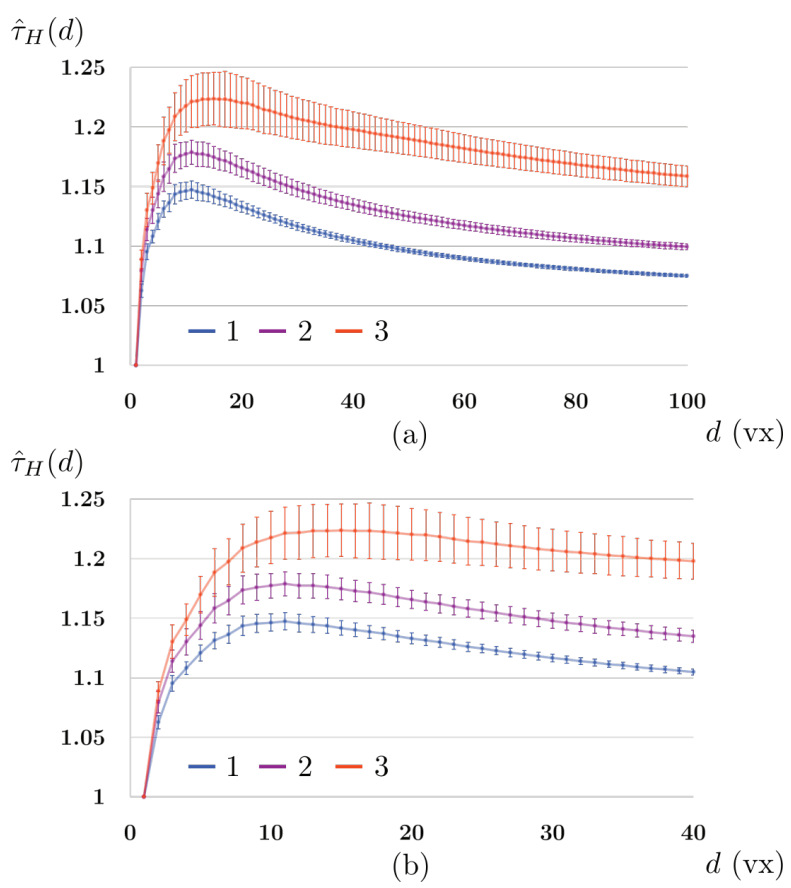

Fig. 14: H-tortuosity curves, $\hat{\tau}_{H}(d)$, as a function of the distance $d$, for the three models considered and defined above (a), and (b) a zoom over local distances. Confidence intervals, with confidence level at $95 \%$, are represented by vertical bars.

The second case is composed of three Cox multiscale Boolean models with $V_{v, O U T}, V_{v, T O T}$ and $R_{I N C}$ 
fixed, equal to $0,0.2$ and 20 , respectively. $V_{v, T O T}$ is defined as

$$
V_{v, T O T}=V_{v} \cdot V_{v, I N C}+V_{v, O U T} \cdot\left(1-V_{v, I N C}\right) .
$$

The different models are then defined by:

1. $V_{v}=0.25$ and $V_{v, I N C}=0.8$,

2. $V_{v}=0.5$ and $V_{v, I N C}=0.4$,

3. $V_{v}=1$ and $V_{v, I N C}=0.2$.

In this case, $\eta$ is equal to the infinity. The three models are defined by $D \eta$ equal to $0.25,0.5$ and 1 , respectively. Fig. 15 shows two realizations of two distinct models, (1) and (3). The indices are the same as the ones used in Fig. 16.

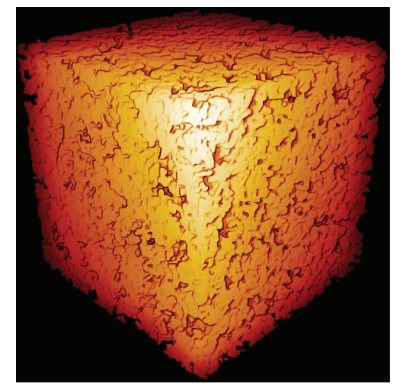

(1)

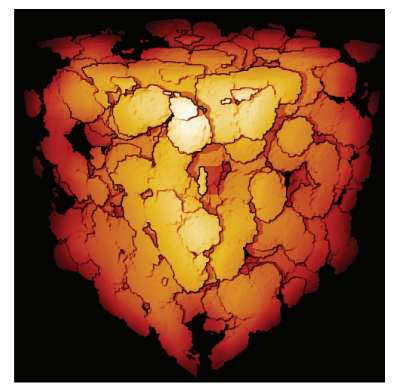

(3)
Fig. 15: Two realizations of two distinct Cox multiscale Boolean models of platelets $(l=L=13$ and $H=3$ ), (1) $V_{v}=0.25, V_{v, I N C}=0.8$ and (3) $V_{v}=1$, $V_{v, I N C}=0.2$. Volumes generated and rendered using "plug'im!" (2018).

The H-tortuosity is applied on each model, the results are presented in Fig. 16. As previously, in Fig. 16(a-b) the higher the imposed geometric heterogeneity, the higher the morphological tortuosity, whatever the distance. Here again, the maximal tortuosity and its corresponding abscissa discriminate the three models. This correlation between tortuosity and heterogeneity, highlighted by the two previous application cases, is in good agreement with the conclusions of Hollewand and Gladden (1995). The stabilization value could probably discriminate the models too, but the stabilization is not reached at $d=$ 100.

The conclusions are pretty much the same. The main difference between the two cases is $V_{v, T O T}$, which increases with geometric heterogeneity in the first case, and is fixed in the second one. This highlights the fact that, geometric heterogeneity has an influence over global characteristics. Indeed, in Fig. 14, we could think that the distinct stabilization values are due only to the various values of $V_{v, T O T}$.
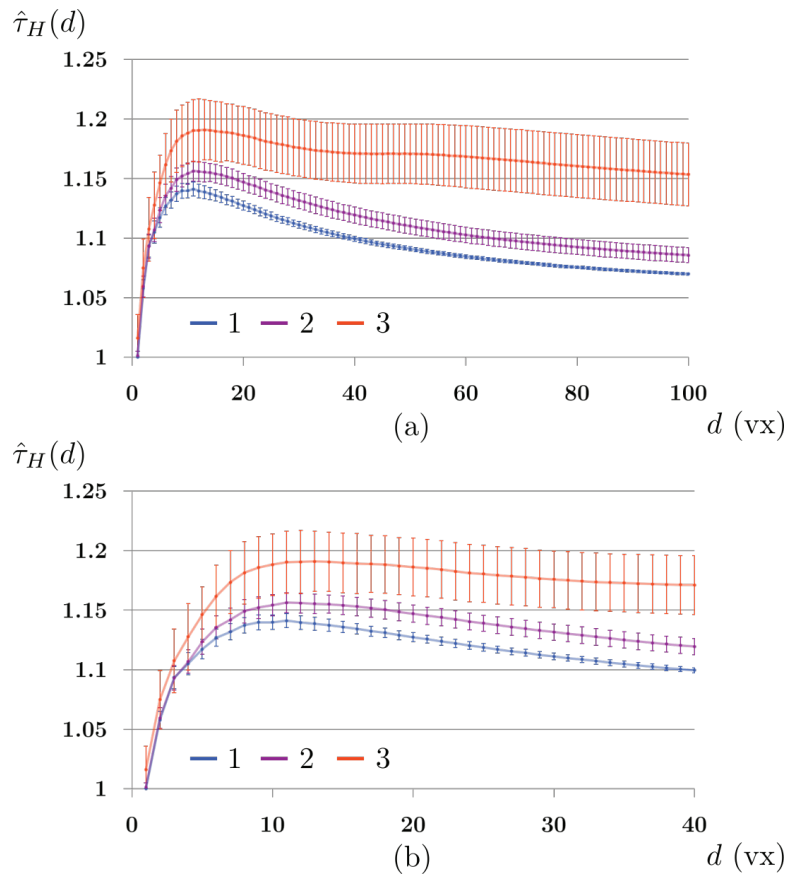

Fig. 16: H-tortuosity curves, $\hat{\tau}_{H}(d)$, as a function of the distance $d$, for the three models considered and defined above (a), and (b) a zoom over local distances. Confidence intervals, with confidence level at $95 \%$, are represented by vertical bars.

Bottleneck effects are then considered for characterization of such models using the $H$-tortuosityby-iterative-erosions. The results are shown in Fig. 17. The display is arbitrarily limited to $r<4$, for readability purposes. This value is sufficient to highlight bottleneck effects.

Considering constrictivity in the local variations of the topology brings additional information. As expected, the H-tortuosity-by-iterative-erosions increases the discriminative power. Indeed, the three models react differently to the iterative erosions. At constant $V_{v, T O T}$ and $V_{v, O U T}$, the more heterogeneous the microstructure, the smaller $V_{v, I N C}$, the less bottleneck effects contribute to an increasing of the average local tortuosities. This is due to the fact that, outside aggregates, bottleneck effects are less.

We can also notice that the confidence intervals increase with $r$, the radius used for the erosions corresponding to the size of the percolating particle. This effect is linked to the concept of representative volume element (RVE) (Hill, 1963; Kanit et al., 2003). Using the duality between morphological erosion and dilation, the erosion of the porous phase is equivalent to the dilation of the solid phase. A looser statement is that a dilated realization can be seen as a realization of the same model but with bigger grains and lower porosity. Consequently, the more eroded the porous phase, the bigger the RVE. This comment also applies to the M-tortuosity-by-iterative-erosions, in 
Fig. 9(b), and is in good agreement with the results of Neumann et al. (2020), showing, among other things, the increase in the variance of the probability density distributions of tortuosity, as a function of the size of the percolating particle.

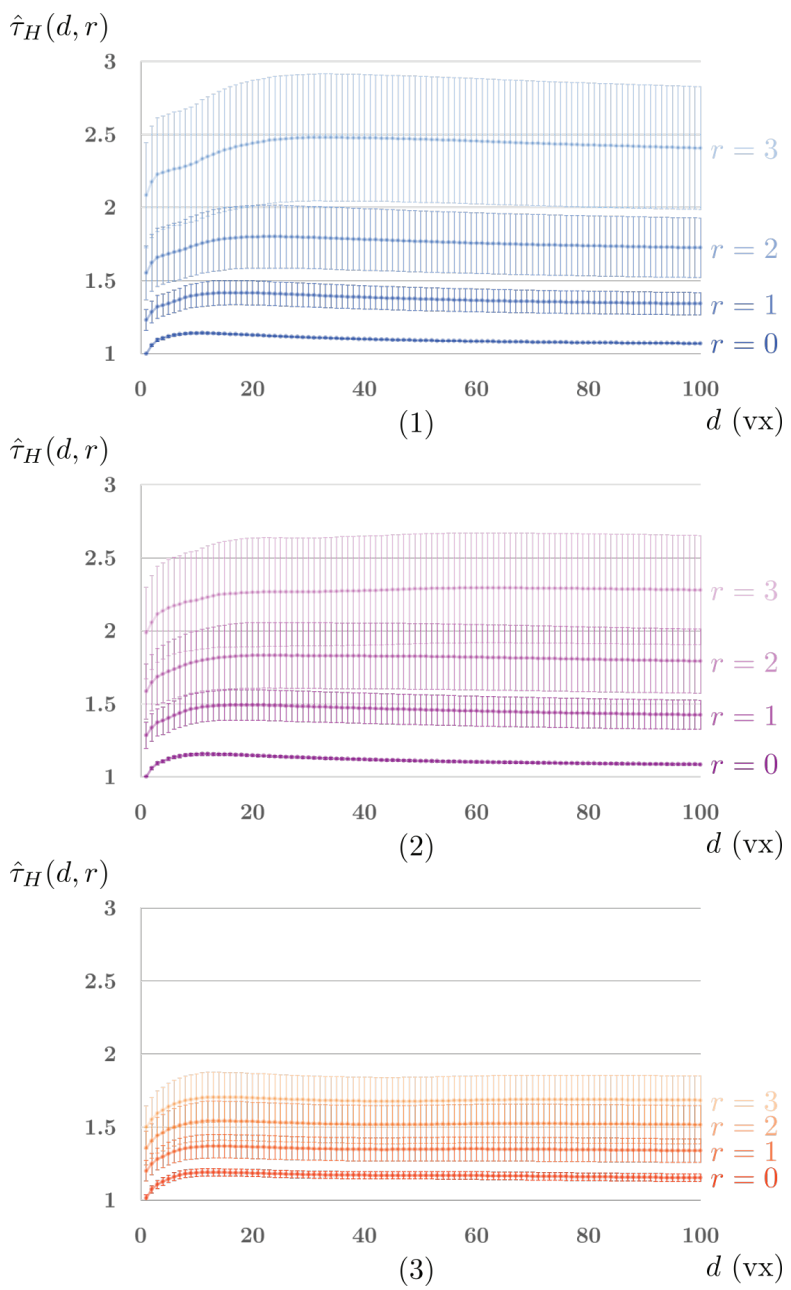

Fig. 17: H-tortuosity-by-iterative-erosions curves, $\hat{\tau}_{H}(d, r)$, as a function of the distance $d$ and the radius $r$, for the three models considered and defined above (1), (2) and (3). Confidence intervals, with confidence level at $95 \%$, are represented by vertical bars.

\section{CONCLUSION AND PERSPECTIVES}

Heterogeneity is a common characteristic of many microstructures, especially for real and industrial porous media. The H-tortuosity, based on the $M$ tortuosity formalism, assesses the impact of geometric heterogeneity over the microstructure topology. For this purpose, the $\mathrm{H}$-tortuosity estimator computes the average variations of the morphological tortuosity as a function of the Euclidean distance, by probing the peripheral microstructures of random points. This descriptor quantifies the scale heterogeneity of the microstructure. Like the M-tortuosity, the $H$ tortuosity can be applied to complex porous networks and manages disconnections. Both descriptors are complementary, which has been highlighted by considering Boolean models of spheres and spherocylinders.

While the M-tortuosity focuses on characterizing the global porous network, the $\mathrm{H}$-tortuosity is committed to the evolution of morphological tortuosity with scale. Cox multi-scale Boolean models of platelets are then used for highlighting the H-tortuosity sensitivity to geometric heterogeneity. Two cases have been presented to validate the descriptor behavior.

The H-tortuosity-by-iterative-erosions is an extension of the H-tortuosity, similar to the M-tortuosity-byiterative-erosions. This second descriptor takes into account bottleneck effects, and assesses the local variations of the microstructure topology as seen by a spherical particle of given size. The H-tortuosity is closely connected to percolation concept and the $H$ tortuosity-by-iterative-erosions is futhermore linked to the constrictivity concept. Its behavior has been validated on the final case of Cox multi-scale Boolean models.

The perspectives of this work are multiple. The theoretical perspectives include comparison with theoretical values and with the state-of-art for morphological tortuosity assessment, analysis of relevant applications, assessment of the limits of use with consideration of extreme cases, and study of the impact of the skeleton, partly done in Chaniot et al. (2019).

There is no difficulty to apply the definitions to random closed sets, and it has been used as such for a long time in previous publications, given in the list of references. The challenge is to provide theoretical estimations of this descriptors for given random sets models. For instance, in Gouéré and Théret (2017) the positivity of the geodesic distance is proved for the Boolean model, in connection to percolation. In Willot (2015), upper bounds of geodesic distances are given in 2D and 3D porous Boolean and multiscale Boolean models, for vanishing pore volume fractions.

Instead, the present paper was focused on algorithms to estimate the morphological tortuosity, from some random samplings of points in the microstructure, and on applications to simulations of some models of random closed sets, like Boolean and Cox multiscale Boolean models.

Finally, extensions of the M-tortuosity and the $H$ tortuosity to the functional case will be defined for the characterization of grayscale images, and with detailed analysis of their behavior.

The logical next step is the application perspectives. 
The original purpose of the definitions of the $M$ tortuosity and the H-tortuosity, is to provide numerical descriptors for the characterization of binary images, representing complex microstructures. The complexity can be seen in the interconnected network itself, but in the nature of the image too, as it is the case for catalysts, imaged using electron tomography (Tran et al., 2014). More specifically, $\gamma$-aluminas will be considered.

This imaging technology provides 3D images with high resolution and very complex structure, i.e. shape of the sample, as shown in Fig. 18. The specificities of our descriptors make the characterization of such numerical microstructures possible. Indeed, defining representative entries and exits in such materials is a delicate task. Our descriptors, based on Monte Carlo method, can handle this issue. Moreover, high resolution for high precision yields big size images, with a long processing time. Our algorithms provide the possibility of computation time efficiency thanks to the unique sampled points set $S$. This choice takes us away from the realm of random variables, but decreases the number of distance maps to be computed, which is the key point to improve computation time efficiency.

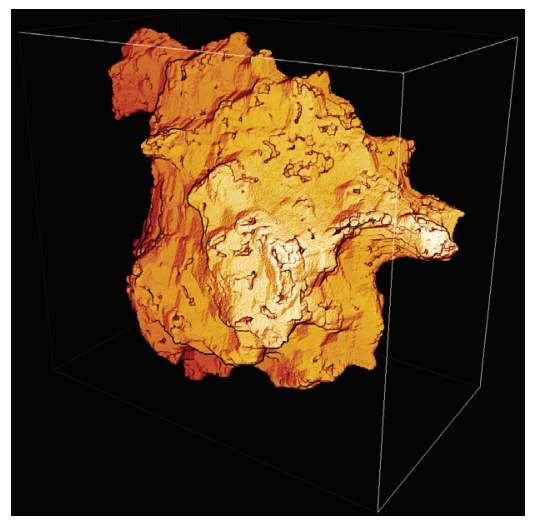

Fig. 18: A sample of a specific $\gamma$-alumina, obtained by electron tomography, reconstructed, filtered, and segmented. The porous volume is in yellow, the solid phase and the surrounding void are transparent.

Finally, a deterministic numerical definition of the $M$-tortuosity is proposed in Batista et al. (2020), where $S$ is imposed and represents the locations of particles at the surface of the catalyst support, a porous medium. A similar extension for the H-tortuosity would allow a more detailed analysis of the impact of structural features on the transport properties.

\section{ACKNOWLEDGEMENTS}

We would like to thank the reviewers for their relevant and constructive comments.

\section{REFERENCES}

Adler PM (1992). Porous media: geometry and transports. Butterworth-Heineman, Boston, MA, 544.

Adler PM, Thovert J-F (1998). Real porous media: Local geometry and macroscopic properties. Appl Mech Rev 51(9):537-585.

Baddeley A, Jensen EBV (2004). Stereology for statisticians. Mg Stat Pro 103:03.

Balberg I, Anderson CH, Alexander S, Wagner N (1984). Excluded volume and its relation to the onset of percolation. Phys Rev B 30(7):3933.

Barman S, Rootzén H, Bolin D (2019). Prediction of diffusive transport through polymer films from characteristics of the pore geometry. AIChE J 65(1), 446-57.

Batista ATF, Baaziz W, Taleb A-L, Chaniot J, Moreaud M, Legens C, Aguilar-Tapia A, Proux O, Hazemann J-L, Diehl F, Chizallet C, Gay A-S, Ersen O, Raybaud P (2020). Atomic scale insight into the formation, size and location of platinum nanoparticles supported on $\gamma$-alumina. ACS Catal 10(7):4193-204.

Berrocal CG, Löfgren I, Lundgren K, Görander N, Halldén C (2016). Characterisation of bending cracks in R/FRC using image analysis. Cement Concrete Res 90:104-16.

Bini F, Pica A, Marinozzi A, Marinozzi F (2019). A 3D Model of the Effect of Tortuosity and Constrictivity on the Diffusion in Mineralized Collagen Fibril. Sci Rep-UK 9(1):2658.

Borgefors G (1986). Distance transformations in digital images. Comput Vision Graph 34(3):34471.

Bortolussi V, Figliuzzi B, Willot F, Faessel M, Jeandin M (2018). Morphological modeling of cold spray coatings. Image Anal Stereol 37(2):145-58.

Caflisch RE (1998). Monte Carlo and quasi-Monte Carlo methods. Acta Numer 7:1-49.

Carman PC (1937). Fluid flow through granular beds. Trans Inst Chem Eng 15:150-66.

Chaniot J, Moreaud M, Sorbier L, Becker J-M, Fournel $T$ (2019). Tortuosimetric operator for complex porous media characterization. Image Anal Stereol 38(1):25-41.

Chiu SN, Stoyan D, Kendall WS, Mecke J (2013). Stochastic geometry and its applications. John Wiley \& Sons.

Clennell MB (1997). Tortuosity: a guide through the maze. Geol Soc Spec Publ 122(1):299-344. 
Criminisi A, Sharp T, Rother C, Pérez P (2010). Geodesic image and video editing. ACM T Graphic 29(5):134-1.

Decker L, Jeulin D, Tovena I (1998). 3D morphological analysis of the connectivity of a porous medium. Acta Stereol 17(1).

Dullien FAL (1979). Porous media: fluid transport and pore structure. Academic press.

Ghanbarian B, Hunt AG, Ewing RP, Sahimi M (2013). Tortuosity in porous media: a critical review. Soil Sci Soc Am J 77(5):1461-77.

Ghanbarian B, Hunt AG, Sahimi M, Ewing RP, Skinner TE (2013). Percolation theory generates a physically based description of tortuosity in saturated and unsaturated porous media. Soil Sci Soc Am J 77(6):1920-29.

Gouéré J-B, Théret M (2017). Positivity of the time constant in a continuous model of first passage percolation. Electron J Probab 22.

Graham D (1957). Geometric heterogeneity in the adsorption of nitrogen on graphitized carbon surfaces. J Phys Chem-US 61(10):1310-13.

Grujicic M, Cao G, Roy WN (2004). A computational analysis of the percolation threshold and the electrical conductivity of carbon nanotubes filled polymeric materials. J Mater Sci 39(14):4441-9.

Hill R (1963). Elastic properties of reinforced solids: some theoretical principles. J Mech Phys Solids 11(5):357-72.

Hollewand MP, Gladden LF (1995). Transport heterogeneity in porous pellets-I. PGSE NMR studies. Chem Eng Sci 50(2):309-26.

Holzer L, Wiedenmann D, Münch B, Keller L, Prestat M, Gasser Ph, Robertson I, Grobéty B (2013). The influence of constrictivity on the effective transport properties of porous layers in electrolysis and fuel cells. J Mater Sci 48(7):2934-52.

Jean A, Jeulin D, Forest S, Cantournet S, N'Guyen F (2011). A multiscale microstructure model of carbon black distribution in rubber. $\mathrm{J}$ Microsc 241(3):243-60.

Jeulin D (1993). Damage simulation in heterogeneous materials from geodesic propagations. Eng Computation 10(1):81-91.

Jeulin D (1996). Modeling heterogeneous materials by random structures. Invited lecture, European Workshop on Application of Statistics and Probabilities in Wood Mechanics, Bordeaux , N06/96/MM, Paris School of Mines Publication.

Jeulin D (1997). Advances in Theory and Applications of Random Sets. In : Advances In Theory And
Applications Of Random Sets: Proceedings Of The Symposium. World Scientific 105.

Jeulin D, Moreaud M (2006). Percolation of multi-scale fiber aggregates. S4G (Stereology, Spatial Statistics and Stochastic Geometry) 6th International Conference, Prague, Czech Republic.

Jeulin D (2010). Multi scale random models of complex microstructures. Mater Sci Forum 638:81-6.

Jeulin D (2012). Morphology and effective properties of multi-scale random sets: A review. CR Mecanique 340(4-5):219-29.

Kanit T, Forest S, Galliet I., Mounoury V., Jeulin D. (2003). Determination of the size of the representative volume element for random composites: statistical and numerical approach. Int J Solids Struct 40(13-14):3647-79.

Karimpouli S, Tahmasebi P (2019). 3D multifractal analysis of porous media using 3D digital images: considerations for heterogeneity evaluation. Geophys Prospect 67(4):1082-93.

Kingman JFC (1993). Poisson Processes. Oxford Science Publications, Oxford Studies in Probability 3.

Lantuéjoul C, Beucher S (1981). On the use of the geodesic metric in image analysis. $\mathrm{J}$ Microsc 121(1):39-49.

Lohou C, Bertrand G (2005). A 3D 6-subiteration curve thinning algorithm based on P-simple points. Discrete Appl Math 151(1):198-228.

Matheron G (1975). Random sets and integral geometry. Wiley New York.

Moreaud M, Chaniot J, Fournel T, Becker J-M, Sorbier L (2018). Multi-scale stochastic morphological models for $3 \mathrm{D}$ complex microstructures. 17th Workshop on Information Optics (WIO), Quebec, Canada, IEEE, 1-3.

Neumann M, Charry, EM, Zojer K, Schmidt V (2020). On variability and interdependence of local porosity and local tortuosity in porous materials: a case study for sack paper. Methodol Comput Appl $1-15$.

Neumann M, Hirsch C, Staněk J, Beneš V, Schmidt V (2019). Estimation of geodesic tortuosity and constrictivity in stationary random closed sets. Scand J Stat 46:848-84.

Neumann M, Abdallah B, Holzer L, Willot F, Schmidt V (2019). Stochastic 3D Modeling of ThreePhase Microstructures for Predicting Transport Properties: A Case Study. Transport Porous Med, $1-22$. 
Newman MEJ, Ziff RM (2001). Fast Monte Carlo algorithm for site or bond percolation. Phys Rev E 64(1):016706.

Neyman J (1934). On the two different aspects of the representative method: the method of stratified sampling and the method of purposive selection. $\mathrm{J}$ R Stat Soc 97(4):558-625.

Ohser J, Ferrero C, Wirjadi O, Kuznetsova A, Duell J, Rack A (2012). Estimation of the probability of finite percolation in porous microstructures from tomographic images. Int J Mater Res 103(2):18491.

Petersen EE (1958). Diffusion in a pore of varying cross section. AIChE J 4(3):343-5.

Peyrega C, Jeulin D (2013). Estimation of tortuosity and reconstruction of geodesic paths in 3D. Image Anal Stereol 32(1):27-43.

Raybaud P, Toulhoat H (2013). Catalysis by transition metal sulphides: From molecular theory to industrial application. Editions Technip.

Rigby SP, Gladden LF (1996). NMR and fractal modelling studies of transport in porous media. Chem Eng Sci 51(10):2263-72.

Rosenfeld A, Pfaltz JL (1968). Distance Functions on Digital Pictures. Pattern Recogn 1:33-61.

Rutovitz D (1968). Data structures for operations on digital images. Pictorial pattern recognition 10533.

Saha PK, Borgefors G, Di Baja GS (2016). A survey on skeletonization algorithms and their applications. Pattern Recogn Lett 76:3-12.

Savary L, Jeulin D, Thorel A (1999). Morphological analysis of carbon-polymer composite materials from thick sections. Acta Stereol 18(3):297-303.
Serra J (1982). Image Analysis and Mathematical Morphology. Academic Press, London.

Toivanen PJ (1996). New geodesic distance transforms for gray-scale images. Pattern Recogn Lett 17(5):437-50.

Tran V-D, Moreaud M, Thiébaut E, Denis L, Becker J-M (2014). Inverse problem approach for the alignment of electron tomographic series. Oil Gas Sci Technol 69(2):279-91.

Van Brakel J, Heertjes PM (1974). Analysis of diffusion in macroporous media in terms of a porosity, a tortuosity and a constrictivity factor. Int J Heat Mass Tran 17(9):1093-103.

Vogel H (2002). Topological characterization of porous media. Morphology of condensed matter 75-92.

Wang H, Pietrasanta A, Jeulin D, Willot F, Faessel M, Sorbier L, Moreaud M (2015). Modelling mesoporous alumina microstructure with 3D random models of platelets. J Microsc 260(3):287301.

Warren JE, Price HS (1961). Flow in heterogeneous porous media. Soc Petrol Eng J 1(3):153-69.

Wernert V, Bouchet R, Denoyel R (2010). Influence of molecule size on its transport properties through a porous medium. Anal Chem 82(7):2668-79.

Willot F (2015) The power laws of geodesics in some random sets with dilute concentration of inclusions. ISMM 2015 535-46.

"plug im!" an open access and customizable software for signal and image processing. https://www.plugim.fr (2018). 